\title{
A 0.8-6 GHz Wideband Receiver Front-End for Software-Defined Radio
}

\author{
Kuan-Ting Lin, ${ }^{1}$ Tao Wang, ${ }^{2}$ and Shey-Shi Lu' \\ ${ }^{1}$ Graduate Institute of Electronics Engineering, National Taiwan University, Taipei 106, Taiwan \\ ${ }^{2}$ Department of Electronics Engineering, Chang Gung University, Taoyuan 333, Taiwan
}

Correspondence should be addressed to Shey-Shi Lu; sslu@ntu.edu.tw

Received 8 October 2013; Accepted 15 November 2013

Academic Editor: Ching Liang Dai

Copyright (C) 2013 Kuan-Ting Lin et al. This is an open access article distributed under the Creative Commons Attribution License, which permits unrestricted use, distribution, and reproduction in any medium, provided the original work is properly cited.

A wideband $(0.8-6 \mathrm{GHz})$ receiver front-end (RFE) utilizing a shunt resistive feedback low-noise amplifier (LNA) and a micromixer is realized in $90 \mathrm{~nm}$ CMOS technology for software-defined radio (SDR) applications. With the shunt resistive feedback and series inductive peaking, the proposed LNA is able to achieve a wideband frequency response in input matching, power gain and noise figure (NF). A micromixer down converts the radio signal and performs single-to-differential transition. Measurements show the conversion gain higher than $17 \mathrm{~dB}$ and input matching (S11) better than $-7.3 \mathrm{~dB}$ from 0.8 to $6 \mathrm{GHz}$. The IIP3 ranges from -7 to $-10 \mathrm{dBm}$, and the NF from 4.5 to $5.9 \mathrm{~dB}$. This wideband receiver occupies $0.48 \mathrm{~mm}^{2}$ and consumes $13 \mathrm{~mW}$.

\section{Introduction}

Software-defined radio was designed to process any signal within a certain bandwidth [1]. For an SDR in $0.8-6 \mathrm{GHz}$ region, it includes signals of GSM, $3 \mathrm{G}$, WLAN, Bluetooth, WiMAX, and GPS applications. Such an idea can be realized by using an ultra-high speed ADC for direct sampling, but the power consumption of the high speed ADC is too large to accept. Relatively, a SDR receiver that down-converts signals before ADC appears to be a more practical approach.

The intuitive SDR receiver topology is to connect frontends of different standards in parallel as shown in Figure 1(a); nevertheless, the chip size of such topology would be too large. A wideband radio [2-6] and a tunable-band radio [79] (see Figure 1(b)) are good candidates for this purpose. The most challenging problem is how to design an LNA and a mixer that meet all the requirements in such a wideband from $800 \mathrm{MHz}$ to $6 \mathrm{GHz}$.

A wideband RFE can be implemented by several circuit structures. Conventional common-gate LNAs feature wide input matching and gain bandwidths [4]. However, the multiple stages required by such circuits for gain and noise flatness can be power hungry. A shunt-shunt feedback LNA followed by a passive mixer $[2,5,6]$ can be an option, but its gain degrades at high frequency due to the large capacitance at its input and output stages. Besides, the trade-off between noise figure and bandwidth remains an issue. Tunable-band receivers switching its frequency with tunable passive devices [7-9] would be promising, except that the size of passive devices is too costly to accept. Designs of mixers can be also challenging for SDR. Passive mixers are widely used for frequency down-conversion. Very large power is needed for LO input to drive these passive mixers which results in power consumption and interference problems.

In order to solve the above issues, a wideband RFE utilizing a resistive feedback LNA and a micromixer is proposed. This LNA adopts the resistive feedback technique and inductive peaking to extend the bandwidth $[10,11]$. The micromixer [12-14] topology is used for down-conversion as well as single-to-differential transition in a wide frequency range without the high $\mathrm{LO}$ signal power requirement. As a result, this RFE accomplishes the wideband, low power, and small size criteria of SDR applications.

\section{Receiver Architecture}

Figure 2 shows the block diagram of the proposed SDR system. The receiver comprises a $0.8-6 \mathrm{GHz}$ wideband LNA, two micromixers, baseband blocks, a frequency divider, and 


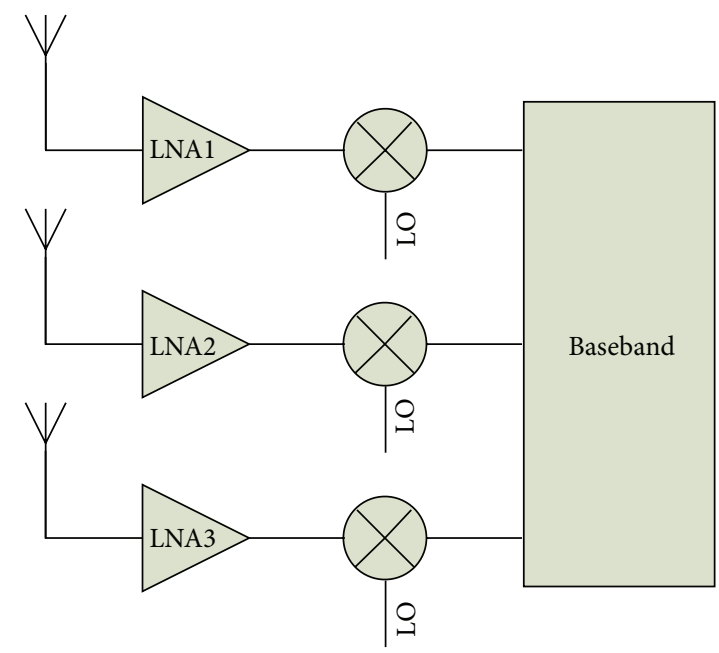

(a)

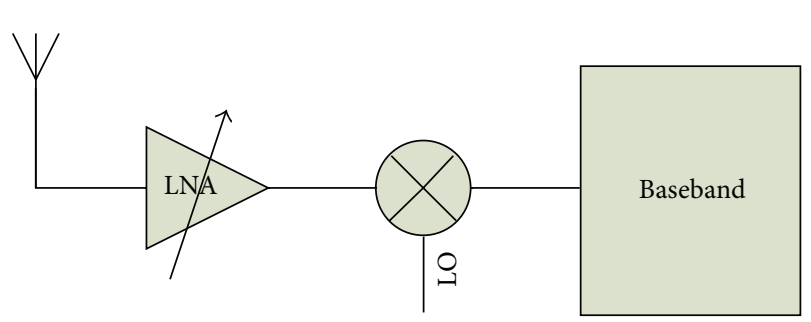

(b)

Figure 1: (a) Multiband receiver architecture. (b) Wideband or tunable-band receiver architecture.

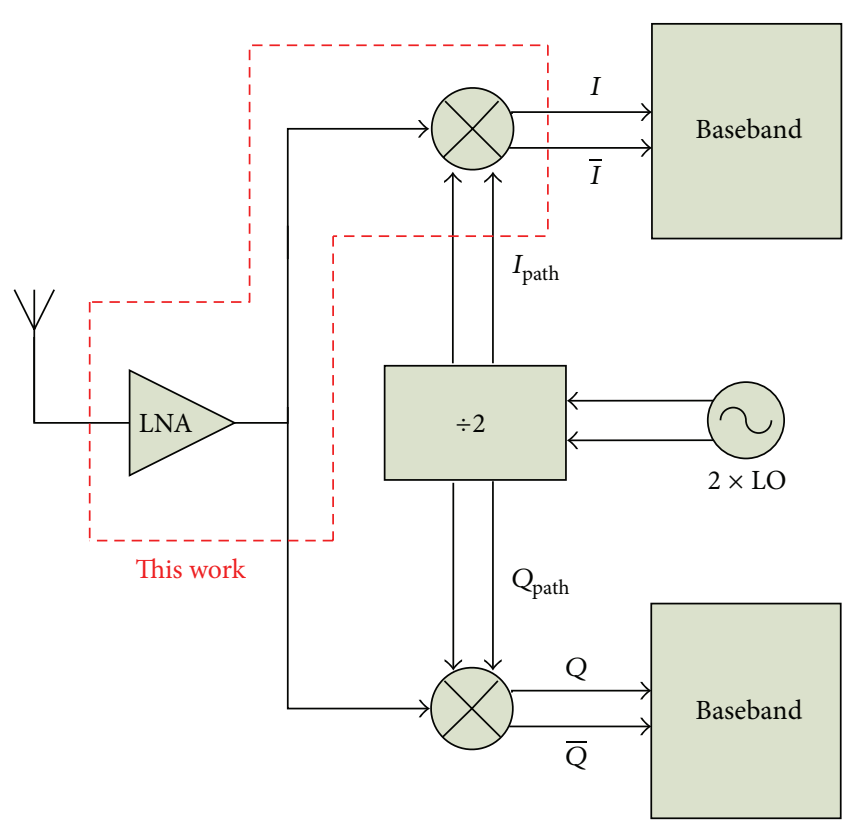

FIGURE 2: Block diagram of SDR receiver.

an LO signal generator. Requirements of the LNA include flat frequency responses of high gain and low NF, wideband matching, and good phase linearity (i.e., small groupdelay variation). After LNA, the I/Q micromixers downconvert signal frequency to a lower band for baseband signal processing. In addition, the frequency divider can generate differential I/Q LO signals.

\section{Design of Wideband Receiver Front-End}

Figure 3 shows the schematic of the proposed RFE. Resistive feedback and inductive peaking technique are adopted in the LNA. The amplified signal is coupled through a capacitor $C_{C}$ to a micromixer. Design principles of each stage are described as follows.
3.1. Wideband LNA. The small-signal model of the proposed LNA is shown in Figure 4, where the $R_{f}$ equal to $\left(R_{\mathrm{FB}}+R_{M}\right) /\left(1+g_{m 1} R_{M}\right)$ is the impedance looking into the input of the LNA after the gate inductor $L_{g}$, and $R_{M}$ equal to $\left(1 / g_{m M 1} / / 1 / g_{m M 2}\right)$ is the input impedance of the micromixer. The input impedance is expressed by (1) with parameters mapping onto Figure 3 . Note that $C_{p}$ represents parasitic capacitance at the input node, and $C_{\text {in }}$ represents the input dc blocking capacitor,

$$
\begin{aligned}
Z_{\text {in }}= & \frac{1}{s C_{\text {in }}} \\
& +\frac{s^{2} R_{f} C_{g s 1} L_{g}+s L_{g}+R_{f}}{s^{3} R_{f} C_{g s 1} C_{p} L_{g}+s^{2} L_{g} C_{p}+s R_{f}\left(C_{g s 1}+C_{p}\right)+1} .
\end{aligned}
$$

As the input impedance of the conventional resistive shuntshunt feedback amplifier, $R_{f}$ provides $50 \Omega$ input matching. Since $C_{\text {in }}$ and $C_{\mathrm{FB}}$ will affect the low frequency matching, they are chosen to be as large as possible $(7 \mathrm{pF}$ and $3 \mathrm{pF}$, resp.). In order to extend the matching range to higher frequencies, a series inductor $L_{g}$ is added to generate a dip in the frequency response of input matching $\left(S_{11}\right)$. The dip frequency is determined by $L_{g}$ and $C_{g s 1}$ values, which are $1.2 \mathrm{nH}$ and $257 \mathrm{fF}$, respectively. The input matching of the LNA can be calculated by substituting (1) into (2),

$$
S_{11}=\frac{Z_{\text {in }}-Z_{0}}{Z_{\text {in }}+Z_{0}} .
$$

The voltage gain of the LNA, $A_{v}$, can be derived and separated by $A_{v i}$ and $A_{v c}$ expressions as illustrated in (3)-(5),

$$
\begin{gathered}
A_{v}=A_{v i} \times A_{v c} \\
A_{v i}=s R_{f} C_{\text {in }}\left(s^{3} L_{g} C_{g s 1} C_{\text {in }} R_{f}+s^{2}\left(L_{g} C_{\text {in }}+R_{f} R_{S} C_{g s 1} C_{\text {in }}\right)\right. \\
\left.+s\left(R_{f} C_{\text {in }}+R_{f} C_{g s 1}+R_{S} C_{\text {in }}\right)+1\right)^{-1},
\end{gathered}
$$




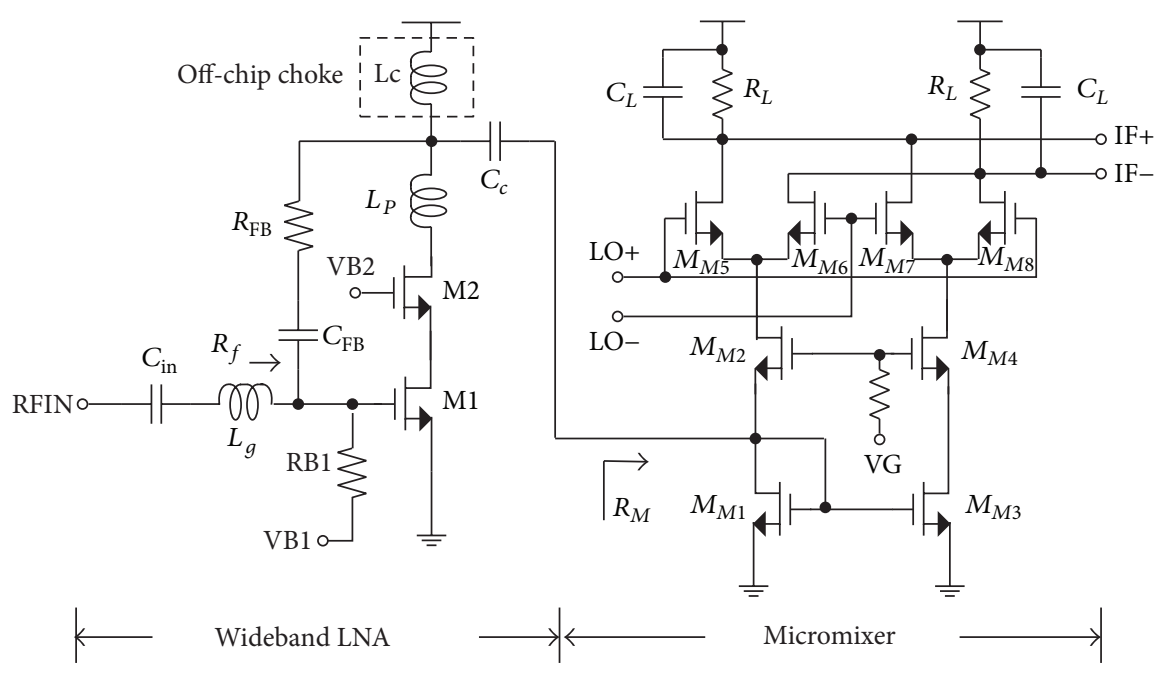

FIGURE 3: Schematic of proposed receiver front-end.

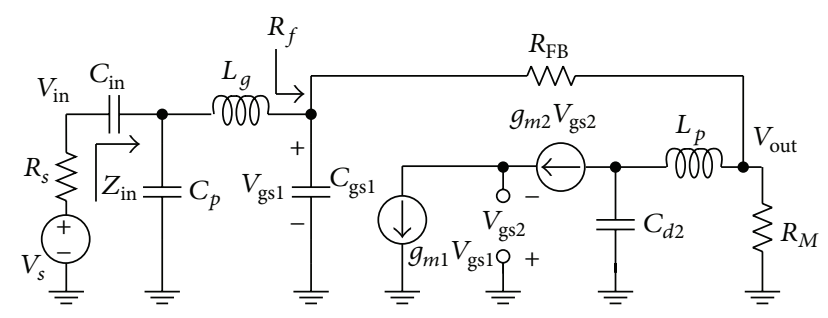

FIGURE 4: Small-signal model of wideband LNA.

$$
A_{v c}=\frac{\left(1 / C_{d 2} L_{p}\right)\left(R_{\mathrm{FB}} / / R_{M}\right)\left(\left(1 / R_{\mathrm{FB}}\right)-g_{m 1}\right)}{s^{2}+s\left(\omega_{0 c} / Q_{c}\right)+\omega_{0 c}^{2}},
$$

where

$$
\begin{gathered}
\omega_{0 c}=\sqrt{\frac{1}{C_{d 2} L_{p}}}, \\
Q_{c}=\frac{1}{R_{\mathrm{FB}} / / R_{M}} \sqrt{\frac{L_{p}}{C_{d 2}}} .
\end{gathered}
$$

$C_{d 2}$ is the capacitance looking into the drain of $M 2$ and $L_{p}$ is the peaking inductor. Since gain starts decreasing at the frequency $1 / 2 \pi\left(R_{\mathrm{FB}} / / R_{M}\right) C_{d 2}$ and the degradation becomes even more serious due to the large size of transistors $M 1$ and $M 2, L_{p}$ is used to resonate out $C_{d 2}$ and extend the bandwidth. The sizes of $M 1$ and $M 2$ are of the same size: 32 fingers with a $W / L$ ratio of $8 \mu \mathrm{m} / 90 \mathrm{~nm}$ per finger. The inductance of $L_{p}$ is $0.55 \mathrm{nH}$.

The noise figure of the LNA is derived according to [11]:

$$
\begin{aligned}
F_{\mathrm{LNA}} \approx & 1 \\
& +\frac{R_{g}+R_{l g}+R_{s s}}{R_{S}}+\frac{\alpha \delta \omega^{2} C_{g s 1}}{5 g_{m 1} R_{S}} \\
& \times\left[\left(R_{S}-\omega^{2} L_{g} R_{S} C_{p}\right)^{2}+\omega^{2} L_{g}^{2}\right]+\frac{R_{\mathrm{FB}}}{R_{S}}
\end{aligned}
$$

$$
\begin{aligned}
\times \mid & \left(s^{2} L_{g}\left(C_{g s 1}+C_{p} g_{m 1} R_{S}\right)\right. \\
& \left.+s\left(m g_{m 1} L_{g}+C_{g s 1} R_{S}\right)+m g_{m 1} R_{S}+1\right) \\
& \times\left.\left(m g_{m 1} R_{\mathrm{FB}}-1\right)^{-1}\right|^{2}+\frac{\gamma g_{m 1}}{\alpha R_{S}} \\
\times \mid & \left(s^{2} L_{g}\left(C_{g s 1}+\frac{C_{p} R_{S}}{R_{\mathrm{FB}}}\right)\right. \\
& \left.+s\left(\frac{L_{g}}{R_{\mathrm{FB}}}+C_{g s 1} R_{S}\right)+\left(\frac{R_{S}}{R_{\mathrm{FB}}}\right)+1\right) \\
& \times\left.\left(g_{m 1}-\frac{1}{m R_{\mathrm{FB}}}\right)^{-1}\right|^{2},
\end{aligned}
$$

where

$$
m \approx \frac{1}{1+s^{2} C_{d 2} L_{p}} .
$$

$R_{S}$ represents the source resistance, $R_{g}$ represents the gate resistance of $M 1, R_{s s}$ represents the source resistance of $M 1$, $R_{l g}$ epresents the series resistance of the gate inductor, $\delta$ and $\gamma$ are the coefficients of gate noise and channel noise, and $\alpha$ is the ratio of $g_{m 1}$ to zero-bias drain conductance $g_{d 0}$.

Note that $R_{\mathrm{FB}}$ affects the input matching, gain, and noise figure. A large $R_{\mathrm{FB}}$ improves the gain and noise performance but results in significant variation across the frequency band. To perform the maximum flat frequency response, a resistance of $340 \Omega$ is used.

Apart from bandwidth extension, the resistive feedback topology improves the linearity of the amplifier [15]. However, the improvement can be observed only in the lower frequency range because of the limited feedback loop bandwidth, as will be seen in the measurement results.

3.2. Micromixer. A micromixer possesses wideband input matching, high linearity, and single-to-differential conversion ability, which makes this topology suitable for the proposed SDR application. 


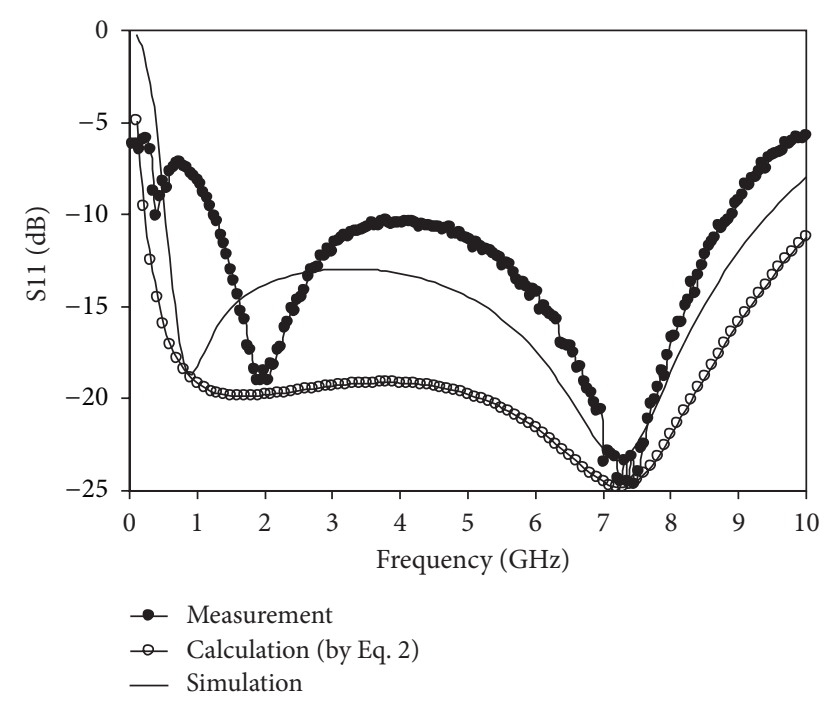

FIGURE 5: Input matching of the proposed receiver front-end.

Single-to-differential conversion is accomplished by injecting the signal current into the source of transistor $M_{M 2}$ and replicating this current to $M_{M 3}$ with the opposite current direction. The sizes of transistors $M_{M 1}-M_{M 4}$ are the same and equals to $48 \mu \mathrm{m} / 90 \mathrm{~nm}$ to balance both paths. Frequency down-conversion is completed by the switching core $M_{M 5}-M_{M 8}$ whose sizes are $90 \mu \mathrm{m} / 90 \mathrm{~nm}$. The output load resistor $R_{L}$ is parallel with $C_{L}$ to filter out high frequency noise and harmonics. $C_{L}(10.8 \mathrm{pF})$ and $R_{L}(500 \Omega)$ generate a pole at $29.5 \mathrm{MHz}$ for sufficient IF bandwidth. To ensure the single-to-differential conversion, the operation frequency of the micromixer should be well below the dominant pole frequency, which is $\omega_{T} / 2$ contributed by $1 / g_{m}$ from $M_{M 1}$ and $2 C_{g s}$ from $M_{M 1}$ and $M_{M 3}$. For a $90 \mathrm{~nm}$ CMOS technology, the cut-off frequency $f_{T}$ is as high as $80 \mathrm{GHz}$; therefore, such topology remains suitable in the interested frequency range.

The conversion gain of the micromixer can be derived as (9), assuming the switching pair operates ideally,

$$
A_{v m}=\frac{2}{\pi}\left(g_{m M 2}+g_{m M 3}\right) R_{L}
$$

The noise factor of the mixer can be obtained by [16] and expressed as follows:

$$
\begin{aligned}
F_{\text {Mixer }}=\frac{\beta}{c^{2}}+(2( & \left.\gamma_{M 3}+r_{g M 3} g_{m}\right) \beta g_{m}+4 \gamma_{M 5} \bar{G} \\
& \left.+4 r_{g M 5} \overline{G^{2}}+\frac{1}{R_{L}}\right)\left(c^{2} g_{m}^{2} R_{S}\right)^{-1}
\end{aligned}
$$

where

$$
\bar{G}=\frac{2 I}{\pi A}
$$

$\beta$ and $c$ are evaluated with the bias current of each switching pair, $\gamma$ and $r_{g}$ are the noise factor and gate resistance of each transistor, $g_{m}$ the transconductance of the $g_{m}$ stage $\left(g_{m}=\right.$ $\left.g_{m M 2}+g_{m M 3}\right)$, and $I$ the current passing through $M 1$ and

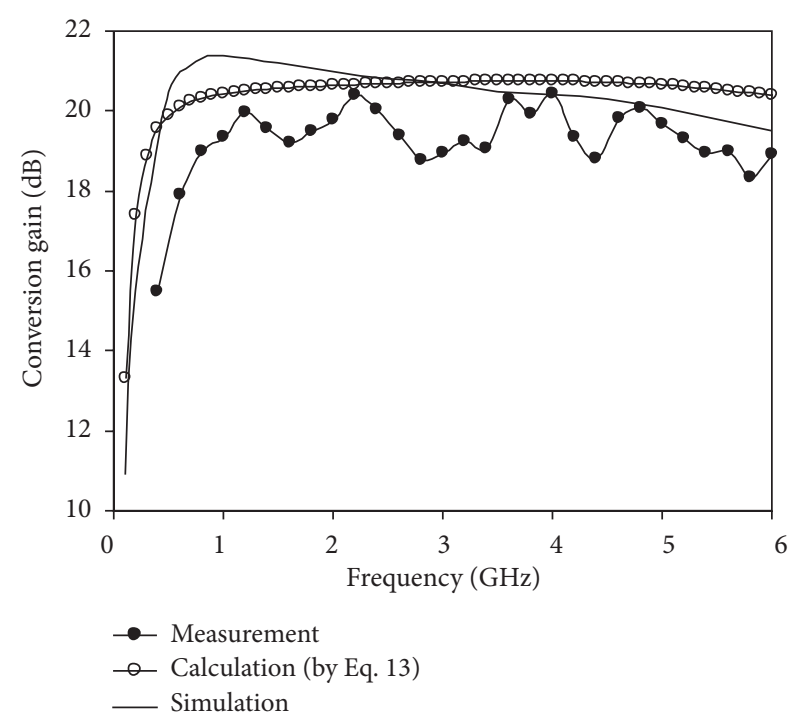

Figure 6: Conversion gain of the proposed receiver front-end.

M3. $A$ is the amplitude of the LO signal and $R_{L}$ is the load resistance of the micromixer. When LO amplitude becomes large enough, $\beta$ and $c$ start to approach 1 and $2 / \pi$, respectively. The effect of the LO port and gate resistance in $g_{m}$ stage is so small that the noise factor can be reduced as (12):

$$
F_{\text {Mixer }} \approx \frac{\beta}{c^{2}}+\frac{2 \gamma_{M 3} g_{m}+4 \gamma_{M 5} \bar{G}+\left(1 / R_{L}\right)}{c^{2} g_{m}^{2} R_{S}} .
$$

The conversion gain of the RFE can be expressed by the product of (3) and (9) and used in the following discussions. Similarly, the noise performance, dominated by LNA, is derived as (14) according to Friis noise formula for further comparisons,

$$
\begin{gathered}
A_{v R X}=A_{v i} \times A_{v c} \times A_{v m}, \\
F_{R X}=F_{\mathrm{LNA}}+\frac{\left(F_{\text {Mixer }}-1\right)}{A_{v}} .
\end{gathered}
$$

\section{Measurement Results and Discussion}

The RFE was realized in 1P9M $90 \mathrm{~nm}$ CMOS technology. On-wafer measurement was performed by using an Agilent 8722ES network analyzer for input matching measurements. Signal generators Agilent E8257D and Agilent E4438C are used to provide LO and RF signals, respectively. Spectrum analyzer Agilent E4440A is used for IF spectrum and noise figure measurements.

Figure 5 shows the measured, calculated, and simulated input matching versus frequency characteristics of the RFE. The $S_{11}$ is below $-10 \mathrm{~dB}$ in $1.2-8.9 \mathrm{GHz}$ and $-7.3 \mathrm{~dB}$ in $0.8-$ $1.2 \mathrm{GHz}$, respectively. The first dip is determined by $R_{f}$ and $C_{\text {in }}$ at low frequency. The second dip is located by $L_{g}$ and $C_{g s 1}$ at high frequency. Figure 6 shows the measured, calculated, and simulated conversion gain versus frequency characteristics of the RFE. The measured conversion gain is larger than $17 \mathrm{~dB}$ from 0.8 to $6 \mathrm{GHz}$ owing to the peaking 
TABLE 1: Summary of the implemented and recently reported state-of-the-art CMOS wideband and tunable-band receiver front-ends.

\begin{tabular}{|c|c|c|c|c|c|c|c|c|c|}
\hline & $\begin{array}{c}\text { Frequency } \\
(\mathrm{GHz})\end{array}$ & $S_{11}(\mathrm{~dB})$ & $\begin{array}{l}\text { Conversion } \\
\text { gain }(\mathrm{dB})\end{array}$ & $\begin{array}{c}\text { Noise } \\
\text { figure }(\mathrm{dB})\end{array}$ & IIP3 (dBm) & $\begin{array}{c}\text { Supply } \\
\text { voltage (V) }\end{array}$ & Power $(\mathrm{mW})$ & Area $\left(\mathrm{mm}^{2}\right)$ & Technology \\
\hline This work & $0.8 \sim 6$ & $<-7.3$ & $17 \sim 20^{*}$ & $4.5 \sim 5.9$ & $-10 \sim-5$ & 1 & $13^{*}$ & $0.48^{*}$ & $90 \mathrm{~nm} \mathrm{CMOS}$ \\
\hline [2] 2008 ISSCC & $0.6 \sim 10$ & $<-10$ & $14^{*}$ & 7 & 0 & 1.2 & $30^{*}$ & $1^{*}$ & $45 \mathrm{~nm}$ CMOS \\
\hline [3] 2007 ISSCC & $2 \sim 8$ & -8 & $23^{*}$ & 4.5 & -7 & 1.2 & $27.8^{*}$ & $0.48^{*}$ & $65 \mathrm{~nm}$ CMOS \\
\hline [4] 2006 JSSC & $0.8 \sim 6$ & N/A & $18 \sim 20^{\#}$ & $5 \sim 5.5$ & -3.5 & 2.5 & $28.5^{*}$ & $3.8^{\$}$ & $90 \mathrm{~nm}$ CMOS \\
\hline [5] 2012 TMTT & $0.6 \sim 3$ & $<-8$ & $42 \sim 48^{\$}$ & 3 & -14 & 1.2 & $30^{\$}$ & $1.5^{\$}$ & $130 \mathrm{~nm}$ CMOS \\
\hline [6] 2011 RFIC & $0.1 \sim 3$ & N/A & $33 \sim 55^{\$}$ & $3.5 \sim 6$ & N/A & 1.2 & $14.5 \sim 48.5^{\$}$ & $2.2^{\$}$ & $130 \mathrm{~nm}$ CMOS \\
\hline [7] 2009 JSSC & $0.1 \sim 5$ & N/A & $-2 \sim 82^{\$}$ & $2.3 \sim 6.5$ & $-10 \sim-3$ & 1.1 & $59 \sim 115^{\$}$ & $2^{\$}$ & $45 \mathrm{~nm}$ CMOS \\
\hline [8] $2008 \mathrm{MWCL}$ & $3 \sim 5$ & $<-7.6$ & $19.8 \sim 24.6^{*}$ & $15.4 \sim 17.4$ & $>-6$ & 2 & $16^{*}$ & $1.14^{*}$ & 0.18 um CMOS \\
\hline [9] 2006 ISSCC & $3 \sim 8$ & N/A & $15 \sim 21^{*}$ & $5 \sim 6.5$ & -2.6 & 2.3 & $44.9^{*}$ & $0.35^{* @}$ & 0.18 um CMOS \\
\hline
\end{tabular}

${ }^{*}$ Front-end; ${ }^{\#}$ LNA only; ${ }^{\$}$ Whole receiver; ${ }^{@}$ active area only.

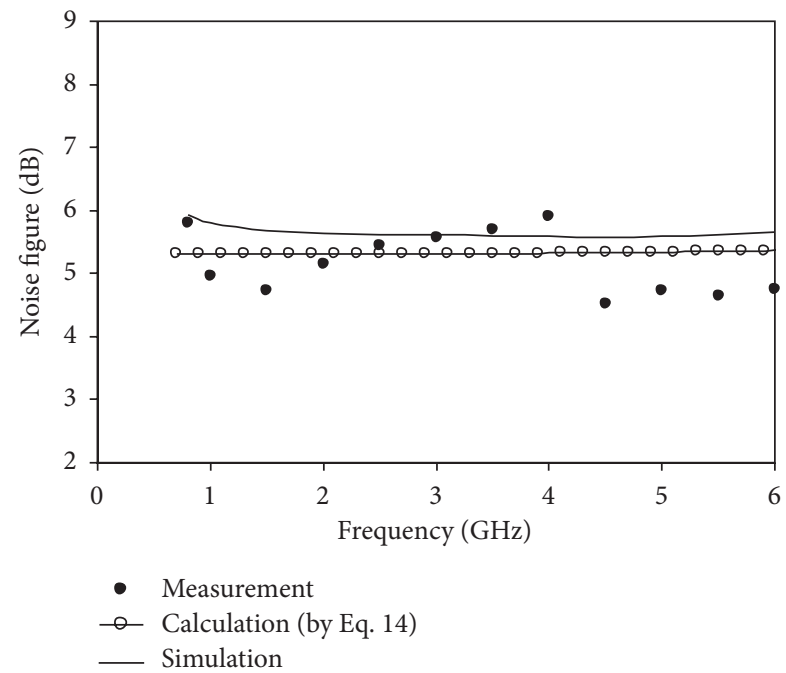

FIGURE 7: Noise figure of the proposed receiver front-end.

inductor $L_{p}$ technique. The declining response at lower frequency is due to the finite value of blocking capacitance $C_{\text {in }}$. Figure 7 shows the measured, calculated, and simulated noise figure versus frequency characteristics of the RFE. The noise figure varies within $5.2 \pm 0.7 \mathrm{~dB}$ in the covered frequency range.

The input third intercept points (IIP3) are in the range from -10 to $-7 \mathrm{dBm}$ over the frequency of interest as shown in Figure 8. As aforementioned, the IIP3 rolls off at higher frequency due to the limited bandwidth of the feedback loop. The power consumption of the LNA and micromixer are 11.5 and $1.5 \mathrm{~mW}$, respectively, under the supply voltage of $1 \mathrm{~V}$. A summary of the implemented and recently reported CMOS wideband and tunable-band RFEs is given in Table 1.

The chip area is $0.48 \mathrm{~mm}^{2}$ including testing pads as shown in Figure 9. The off-chip choke inductor $L_{C}$ can be implemented by integrated passive device (IPD) technique [17] for future integration. In this work, the inductor $L_{C}$ is replaced by a bias-T to demonstrate the RFE performance.

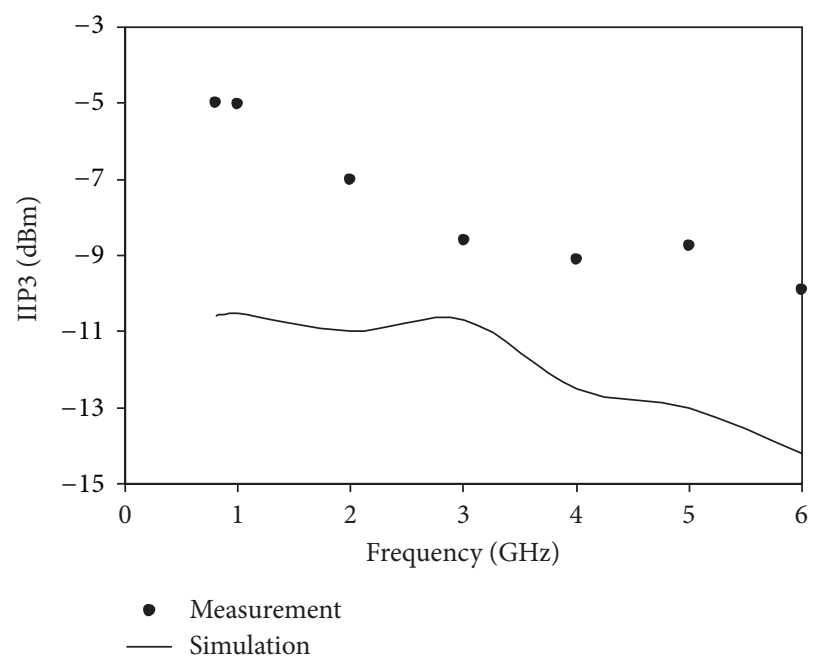

FIGURE 8: IIP3 of the proposed receiver front-end.

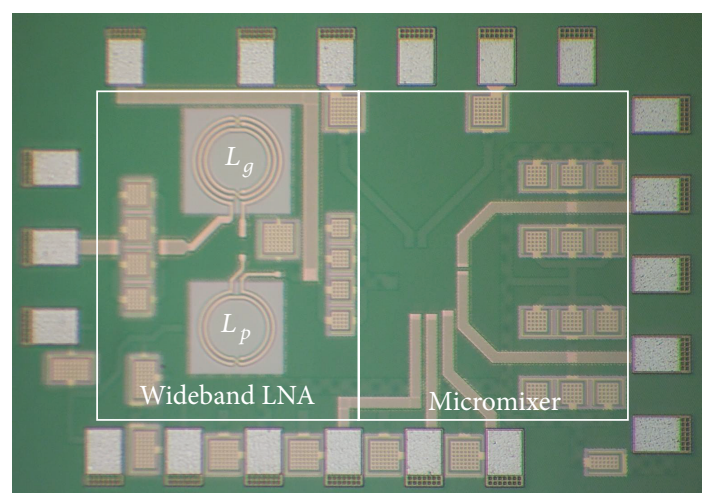

FIgURE 9: Chip micrograph.

\section{Conclusion}

A wideband RFE from 0.8 to $6 \mathrm{GHz}$ is proposed by using a resistive feedback amplifier and a micromixer. The measurement results show a flat and wideband feature in input matching, gain, and noise performance. The proposed RFE 
features the lowest power consumption ( $13 \mathrm{~mW})$ among recently reported silicon-based RFEs in $0.8-6 \mathrm{GHz}$ range.

\section{Acknowledgments}

The authors are very grateful to United Microelectronics Corporation (UMC), Hsin-Chu, Taiwan, for chip fabrication, and National Nano-Device Laboratory (NDL), Hsin-Chu, Taiwan, for their technical supports. This work was supported by the National Science Council under Grant NSC-98-2221E-002-155-MY2.

\section{References}

[1] J. Mitola, "The software radio architecture," IEEE Communication Magazine, vol. 33, no. 5, pp. 26-38, 1995.

[2] R. Van De Beek, J. Bergervoet, H. Kundur, D. Leenaerts, and G. Van Der Weide, "A 0.6-to-10GHz receiver front-end in $45 \mathrm{~nm}$ CMOS," in Proceedings of the IEEE International Solid State Circuits Conference (ISSCC '08), pp. 128-129, February 2008.

[3] S. Lee, J. Bergervoet, K. S. Harish et al., "A broadband receive chain in 65nm CMOS," in Proceedings of the 54th IEEE International Solid-State Circuits Conference (ISSCC '07), pp. 418-419, February 2007.

[4] R. Bagheri, A. Mirzaei, S. Chehrazi et al., "An 800-MHz-6-GHz software-defined wireless receiver in 90-nm CMOS," IEEE Journal of Solid-State Circuits, vol. 41, no. 12, pp. 2860-2875, 2006.

[5] X. Wang, J. Sturm, N. Yan, X. Tan, and H. Min, "0.6-3-GHz wideband receiver $\mathrm{RF}$ front-end with a feedforward noise and distortion cancellation resistive-feedback LNA," IEEE Transactions on Microwave Theory and Techniques, vol. 60, no. 2, pp. 387-392, 2012.

[6] M. Cao, B. Chi, C. Zhang, and Z. Wang, "A 1.2V 0.1-3GHz software-defined radio receiver front-end in 130nm CMOS," in Proceedings of the IEEE Radio Frequency Integrated Circuits Symposium (RFIC '11), June 2011.

[7] V. Giannini, P. Nuzzo, C. Soens et al., "A 2-mm2 0.1-5 GHz software-defined radio receiver in 45-nm digital CMOS," IEEE Journal of Solid-State Circuits, vol. 44, no. 12, pp. 3486-3498, 2009.

[8] C.-R. Wu, H.-H. Hsieh, L.-S. Lai, and L.-H. Lu, "A 3-5 GHz frequency-tunable receiver frontend for multiband applications," IEEE Microwave and Wireless Components Letters, vol. 18, no. 9, pp. 638-640, 2008.

[9] M. Ranjan and L. Larson, "A sub-1mm2 dynamically tuned CMOS MB-OFDM 3-to-8GHz UWB receiver front-end," in Proceedings of the IEEE International Solid-State Circuits Conference (ISSCC '06), pp. 438-445, February 2006.

[10] H.-K. Chen, D.-C. Chang, Y.-Z. Juang, and S.-S. Lu, "A compact wideband CMOS low-noise amplifier using shunt resistivefeedback and series inductive-peaking techniques," IEEE Microwave and Wireless Components Letters, vol. 17, no. 8, pp. 616-618, 2007.

[11] H.-K. Chen, Y.-S. Lin, and S.-S. Lu, "Analysis and design of a 1.628-GHz compact wideband LNA in 90-nm CMOS using a $\pi$ match input network," IEEE Transactions on Microwave Theory and Techniques, vol. 58, no. 8, pp. 2092-2104, 2010.

[12] S.-C. Tseng, C. Meng, C.-H. Chang, C.-K. Wu, and G.-W. Huang, "Monolithic broadband gilbert micromixer with an integrated marchand Balun using standard silicon IC process," IEEE
Transactions on Microwave Theory and Techniques, vol. 54, no. 12, pp. 4362-4371, 2006.

[13] C. Meng, T.-H. Wu, T.-H. Wu, and G.-W. Huang, "A 5.2 GHz 16 $\mathrm{dB}$ gain CMFB Gilbert downconversion mixer using $0.35 \mu \mathrm{m}$ deep trench isolation SiGe BiCMOS technology," in Proceedings of the IEEE MITT-S International Microwave Symposium Digest, pp. 975-978, June 2004.

[14] F. Piazza and Q. Huang, "A high linearity, single-ended input double-balanced mixer in $0.25 \mu \mathrm{m}$ CMOS," in Proceedings of the ESSCIRC (ESSCIRC '12), September 1998.

[15] B. G. Perumana, J.-H. C. Zhan, S. S. Taylor, B. R. Carlton, and J. Laskar, "Resistive-feedback CMOS low-noise amplifiers for multiband applications," IEEE Transactions on Microwave Theory and Techniques, vol. 56, no. 5, pp. 1218-1225, 2008.

[16] M. T. Terrovitis and R. G. Meyer, "Noise in current-commutating CMOS mixers," IEEE Journal of Solid-State Circuits, vol. 34, no. 6, pp. 772-783, 1999.

[17] H.-K. Chen, Y.-C. Hsu, T.-Y. Lin, D.-C. Chang, Y.-Z. Juang, and S.-S. Lu, "CMOS wideband LNA design using integrated passive device," in Proceedings of the IEEE MTT-S International Microwave Symposium (IMS '09), pp. 673-676, June 2009. 

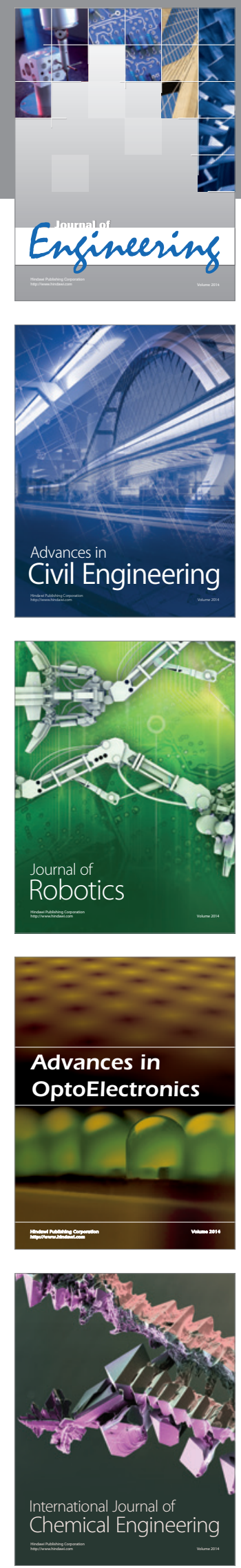

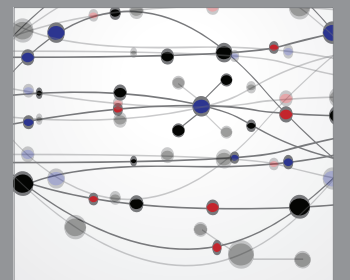

The Scientific World Journal
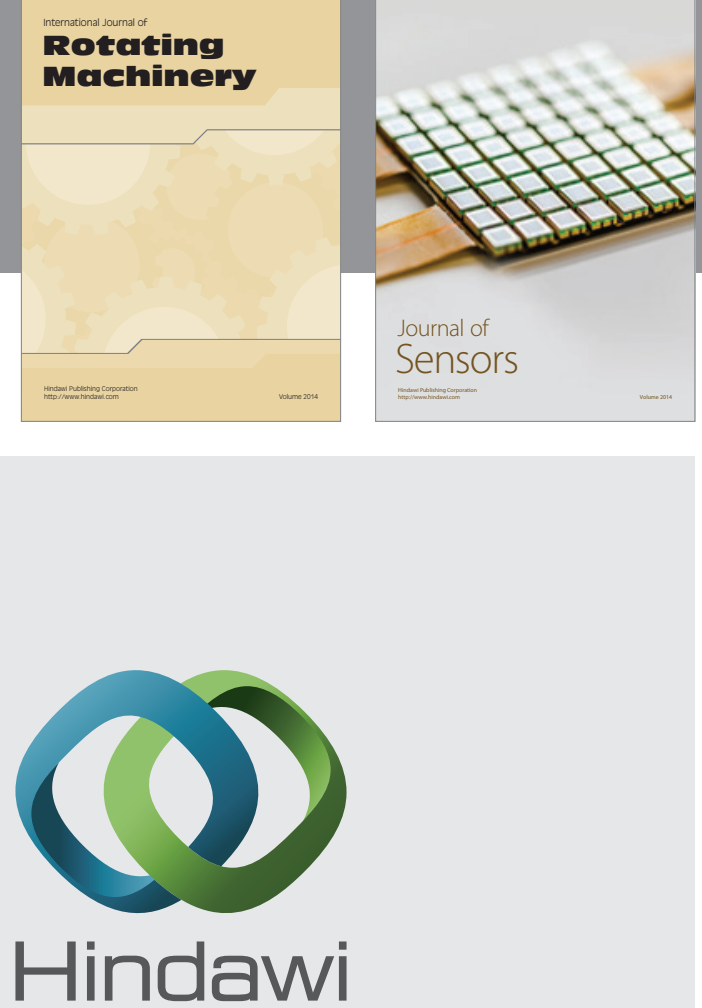

Submit your manuscripts at http://www.hindawi.com
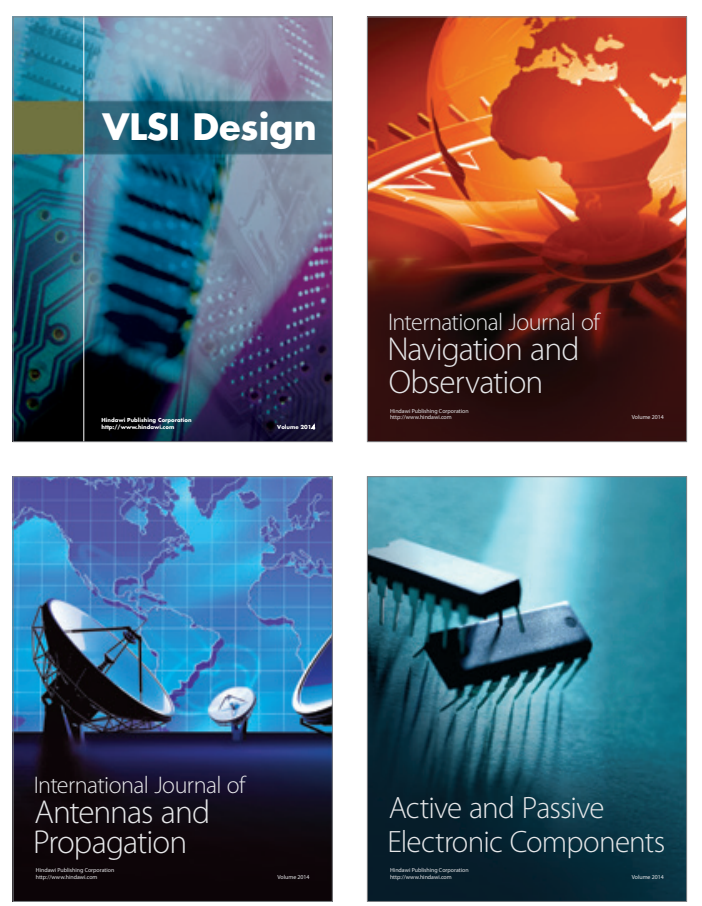
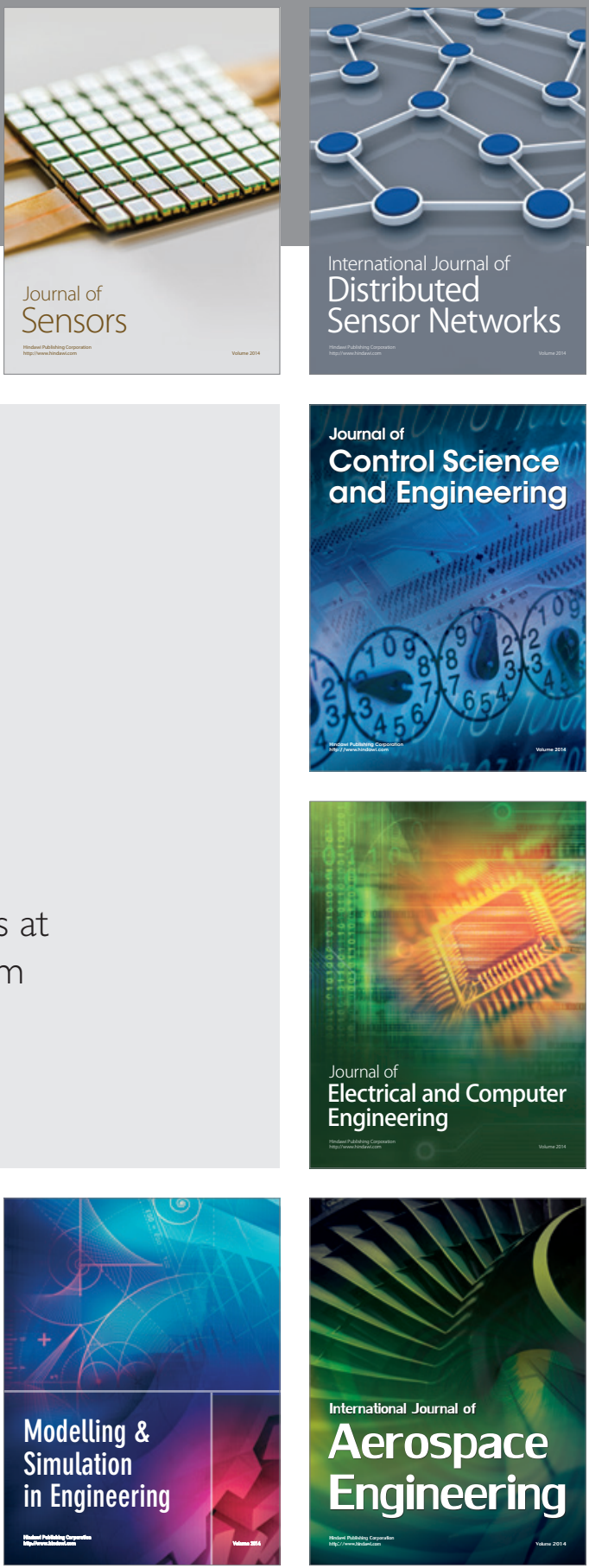

Journal of

Control Science

and Engineering
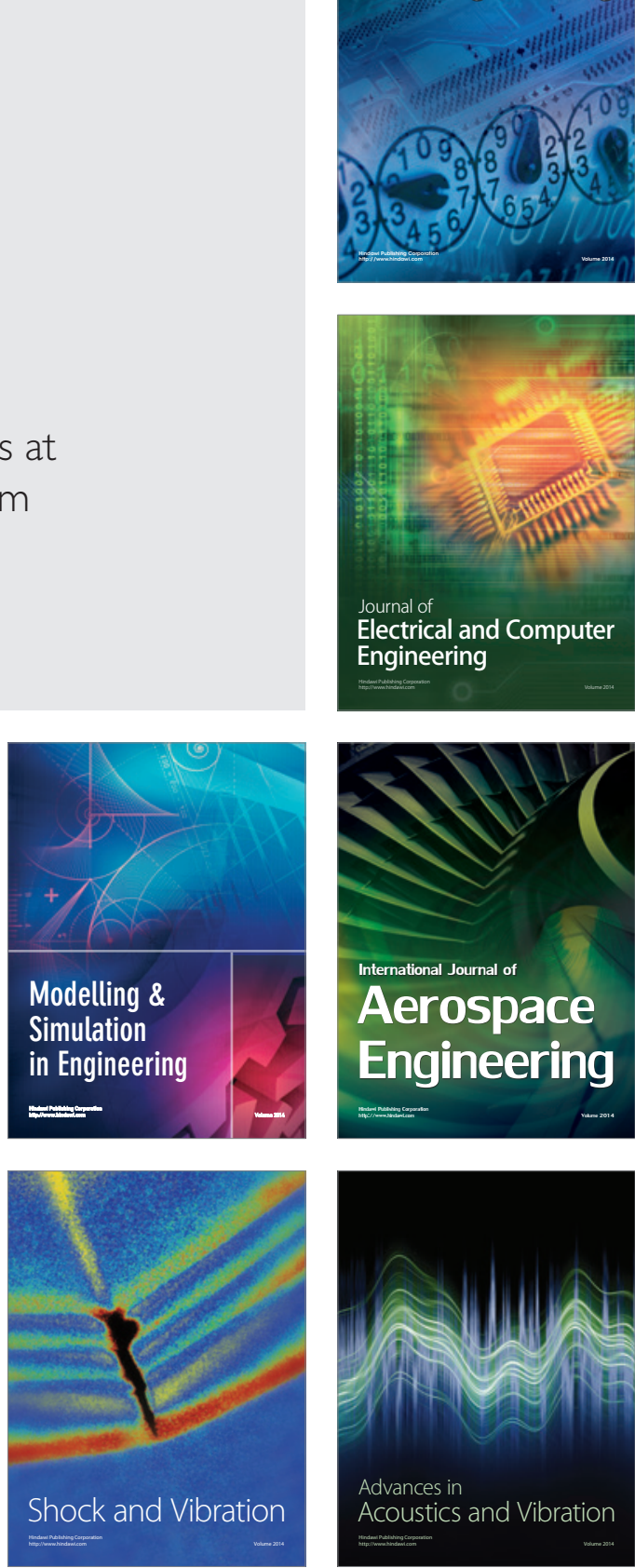